\title{
Soft Tritopological Spaces
}

\author{
Asmhan Flieh Hassan \\ Department of Mathematics, faculty of education for girls, \\ University of Kufa, Iraq
}

\begin{abstract}
The concept of Soft Tritopological Space is introduced in this paper, which we defined it over an initial universe with a fixed set of parameters. Also some new definitions of soft open sets in soft tritopological spaces are introduced and investigated, which are called soft $\tau_{1} \tau_{2} \tau_{3}$-open set, soft $\tau_{1} \tau_{2} \tau_{3}$-pre-open set, soft $\tau_{1} \tau_{2} \tau_{3}$ - $\alpha$-open set (soft tri- $\alpha$-open set) and soft $\delta^{*}$-open set. Consequently we can say that the soft tritopological spaces are more comprehensive and generalized than the classical tritopological spaces.
\end{abstract}

\section{General Terms}

\section{AMS Classification: 54A05, 54A10, 54E55, 54E99.}

\section{Keywords}

Soft set, Soft topological space, Soft bitopological space, Soft tritopological space, soft $\tau_{1} \tau_{2} \tau_{3}$-open set, soft $\tau_{1} \tau_{2} \tau_{3}$-pre-open set, soft $\tau_{1} \tau_{2} \tau_{3}$ - $\alpha$-open set (or soft tri- $\alpha$-open set), soft $\delta^{*}$-open set.

\section{INTRODUCTION}

Soft set theory, initiated in 1999 by Molodtsov [1], he defined the concept of soft set theory as a new mathematical tool, and presented the fundamental results of the new theory and successfully applied it to several directions such as smoothness of functions, theory of probability, operations research, Riemann-integration, Perron integration, etc. A soft set is a collection of approximate descriptions of an object. He also showed how soft set theory is free from the parametrization inadequacy syndrome of fuzzy set theory, rough set theory, probability theory and game theory. Some important applications of soft sets are in information systems and decision making problems can be seen [2][3]. These concepts are of utmost importance in computer science and artificial intelligence. Algebraic structures of soft sets have been discussed in [4], [5], [6].

Concept of Soft topological spaces is introduced in [7], where soft separation axioms have been studied as well. Further contributions to the same concepts have been added by many authors in [8], [9], [10].

In 1963, J.C. Kelly [11], first initiated the concept of bitopological spaces. He defined a bitopological space to set with two topologies and initiated the systematic study of bitopological spaces. In later years, many researchers studied bitopological spaces [12], [13], [14], [15], [16], [17] due to the richness of their structure and potential for carrying out a wide scope for the generalization of topological results in bitopological environment. In 2014, Basavaraj M. Ittanagi [18] introduced the concept of Soft bitopological spaces.

which is defined over an initial universal set with fixed set of parameters, also he introduced some types of soft separation axioms in soft bitopological spaces. A study of soft bitopological spaces is a generalization of the study of soft topological spaces. And in 2016, The Theory of Soft
Ditopological Spaces was presented by G. Senel[19], also T. S. Dizman, A. Sostak and S. Yuksel presented the Soft Ditopological Spaces in the same year[20].

In 2000 Martin M. Kovar.[21], first initiated the concept of tritopological spaces by modify $\theta$-regularity for spaces with two and three topologies. Also in 2003 Luay A. [22], has been initiated the systematic study of tritopological spaces and dealt with in detail and clear. Where they define it as a spaces equipped with three topologies, i.e. triple of topologies on the same set. In 2004, the author Asmhan F. [23] introduce the definition of $\delta^{*}$-open set in tritopological spaces.

In the present paper, concept of soft topological spaces have been generalized to initiate the study of soft tritopological spaces. In addition, we introduce and characterize a new types of soft open sets in a soft tritopological spaces namely soft $\tau_{1} \tau_{2} \tau_{3}$-open set, soft $\tau_{1} \tau_{2} \tau_{3}$-pre-open set, soft $\tau_{1} \tau_{2} \tau_{3}$ - $\alpha$-open set (or soft tri- $\alpha$-open set), soft $\delta^{*}$-open set. And investigate some basic properties.

In section 2, some preliminary concepts about soft topological spaces, bitopological spaces, soft bitopological spaces and tritopological spaces are given. The main section of the manuscript is third which the definition of soft tritopological spaces with examples and some theorems are given. Section 4 is devoted for the definitions of some types of soft open sets in soft tritopological spaces with some examples. Finally in section 5 the conclusions and some ideas of future work is suggested.

This paper is just a beginning of a new structure and we have studied a few ideas only, it will be necessary to carry out more theoretical research to establish a general framework for the practical application.

\section{PRELIMINARIES}

2.1 Definition ([1]) Let $\mathcal{U}$ be an initial universe and $E$ be a set of parameters. Let $\mathcal{P}(\mathcal{U})$ denotes the power set of $\mathcal{U}$ and $\mathcal{A}$ be a non-empty subset of $E$. A pair $(\mathcal{F}, \mathcal{A})$ is said to be a soft set over $\mathcal{U}$, where $\mathcal{F}$ is a mapping given by $\mathcal{F}: \mathcal{A} \rightarrow \mathcal{P}(\mathcal{U})$. In other words, a soft set over $\mathcal{U}$ is a parametrized family of subsets of the universe $\mathcal{U}$. For $\varepsilon \in \mathcal{A}, \mathcal{F}(\varepsilon)$ may be considered as the set of $\varepsilon$-approximate elements of the soft set $(\mathcal{F}, \mathcal{A})$, and if $\varepsilon \notin \mathcal{A}$, then $\mathcal{F}(\varepsilon)=\Phi$,

i.e., $(\mathcal{F}, \mathcal{A})=\{(\varepsilon, \mathcal{F}(\varepsilon)): \varepsilon \in \mathcal{A} \subseteq E, \mathcal{F}: \mathcal{A} \rightarrow \mathcal{P}(\mathcal{U})\}$, Clear that, a soft set is not a set.

2.2 Definition ([6]) The complement of a soft set $(\mathcal{F}, \mathcal{A})$ is denoted by $(\mathcal{F}, \mathcal{A})^{c}$ and is defined by $(\mathcal{F}, \mathcal{A})^{c}=\left(\mathcal{F}^{c}, \neg \mathcal{A}\right)$ where $\mathcal{F}^{c}: \neg \mathcal{A} \rightarrow \mathcal{P}(\mathcal{U})$ is a mapping given by $\mathcal{F}^{c}(\eta)=\mathcal{U}-$ $\mathcal{F}(\eta)$ for all $\eta \in \neg \mathcal{A}$.

Let us call $\mathcal{F}^{c}$ to be the soft complement function of $\mathcal{F}$. Clear that $\mathcal{F}^{c^{c}}$ is the same as $\mathcal{F}$, and $\left((\mathcal{F}, \mathcal{A})^{c}\right)^{c}=(\mathcal{F}, \mathcal{A})$. 
2.3 Definition ([6]) The relative complement of a soft set $(\mathcal{F}, \mathcal{A})$ is denoted by $(\mathcal{F}, \mathcal{A})^{c}$ and is defined by $(\mathcal{F}, \mathcal{A})^{c}=$ $\left(\mathcal{F}^{c}, \mathcal{A}\right)$ where $\mathcal{F}^{c}: \mathcal{A} \rightarrow \mathcal{P}(\mathcal{U})$ is a mapping gives by $\mathcal{F}^{c}(\Omega)=\mathcal{U}-\mathcal{F}(\Omega)$ for all $\Omega \in \mathcal{A}$.

2.4 Definition ([6]) For two soft sets $(\mathcal{F}, \mathcal{A})$ and $(\mathrm{G}, \mathcal{B})$ over a common universe $\mathcal{U}$, we say that $(\mathcal{F}, \mathcal{A})$ is a soft subset of $(\mathrm{G}, \mathcal{B})$ if :

(1) $\mathrm{A} \subseteq \mathrm{B}$.

(2) $\mathrm{F}(\mathrm{e}) \subseteq \mathrm{G}(\mathrm{e})$, for all $\mathrm{e} \in \mathrm{A}$.

$(\mathcal{F}, \mathcal{A})$ is said to be a soft super set of $(\mathrm{G}, \mathcal{B})$, if $(\mathrm{G}, \mathcal{B})$ is a soft subset of $(\mathcal{F}, \mathcal{A})$.

2.5 Definition ([6]) The Union of two soft sets $(\mathcal{F}, \mathcal{A})$ and $(\mathrm{G}, \mathcal{B})$ over the common universe $\mathcal{U}$ is the soft set $(\mathcal{H}, \mathcal{C})$ where $\mathcal{H}(\varepsilon)=\mathcal{F}(\varepsilon) \cup \mathrm{G}(\varepsilon)$ for all $\varepsilon \in E$. We write $(\mathcal{F}, \mathcal{A}) \cup(\mathrm{G}, \mathcal{B})=(\mathcal{H}, \mathcal{C})$

2.6 Definition ([24])The intersection of two soft sets $(\mathcal{F}, \mathcal{A})$ and $(\mathrm{G}, \mathcal{B})$ over the common universe $\mathcal{U}$ is the soft set $(\mathcal{H}, \mathcal{C})$ where $\mathcal{H}(\varepsilon)=\mathcal{F}(\varepsilon) \cap \mathrm{G}(\varepsilon)$ for all $\varepsilon \in E$. We write $(\mathcal{F}, \mathcal{A}) \cap(\mathrm{G}, \mathcal{B})=(\mathcal{H}, \mathcal{C})$

2.7 Definition ([7]) Let $(\mathcal{F}, \mathcal{A})$ be a soft set over $\mathcal{U}$ and $x \in \mathcal{X}$. We say that $x \in(\mathcal{F}, \mathcal{A})$ read as $x$ belongs to the soft set $(\mathcal{F}, \mathcal{A})$ whenever $x \in \mathcal{F}(\lambda)$ for all $\lambda \in E$. Note that for any $x \in \mathcal{X}, x \notin(\mathcal{F}, \mathcal{A})$, if $x \notin \mathcal{F}(\lambda)$ for some $\lambda \in E$.

2.8 Definition ([7]) Let $\tau$ be the collection of soft sets over $\mathcal{X}$, then $\tau$ is said to be a soft topology on $\mathcal{X}$, if :

(1) $\Phi, \mathcal{X}$ belong to $\tau$.

(2) the union of any number of soft sets in $\tau$ belongs to $\tau$.

(3) the intersection of any two soft sets in $\tau$ belongs to $\tau$.

The triple $(\mathcal{X}, \tau, E)$ is called a soft topological space over $\mathcal{X}$.

2.9 Definition ([7]) Let $(\mathcal{X}, \tau, E)$ be a soft topological space over $\mathcal{X}$, then the members of $\tau$ are said to be soft open sets in $x$.

2.10 Definition ([7]) Let $(\mathcal{X}, \tau, E)$ be a soft topological space over $\mathcal{X}$. A soft open set $(\mathcal{F}, E)$ over $\mathcal{X}$ is said to be a soft closed set in $\mathcal{X}$, if its relative complement $(\mathcal{F}, E)^{c}$ belongs to $\tau$.

2.11 Definition([8]) If $(\mathcal{F}, E)$ is a soft set in a soft topological space $(\mathcal{X}, \tau, E)$, then:

(i) The soft closure of $(\mathcal{F}, E)$, denoted by $\operatorname{s.} \operatorname{cl}(\mathcal{F}, E)$ is defined by:

$\operatorname{s.cl}(\mathcal{F}, E)=\bigcap\{(\mathcal{C}, E):(\mathcal{F}, E) \subseteq(\mathcal{C}, E)$, and $(\mathcal{C}, E)$ is soft closed $\}$

(ii) The soft interior of $(\mathcal{F}, E)$, denoted by $\operatorname{sint}(\mathcal{F}, E)$ is defined by: $\operatorname{s.} \operatorname{int}(\mathcal{F}, E)=\bigcup\{(\mathcal{B}, E):(\mathcal{B}, E) \subseteq(\mathcal{F}, E)$, $\operatorname{and}(\mathcal{B}, E)$ is soft open $\}$

2.12 Definition ([11]) Let $X \neq \emptyset, T_{1}$ and $T_{2}$ be two topologies on $X$. Then $\left(X, T_{1}, T_{2}\right)$ is called a bitopological space.

2.13 Definition ([18]) Let $\left(X, \tau_{1}, E\right)$ and $\left(X, \tau_{2}, E\right)$ be the two soft topological spaces over $\mathcal{X}$. Then $\left(\mathcal{X}, \tau_{1}, \tau_{2}, E\right)$ is called a soft bitopological space.

2.14 Definition ([25]) Let $\left(\mathcal{X}, \tau_{1}, \tau_{2}, E\right)$ be a soft bitopological space and a soft set $(\mathcal{F}, E)$ is called soft $\tau_{1} \tau_{2}$-open set if $(\mathcal{F}, E)=(\mathcal{C}, E) \cup(\mathcal{B}, E)$, where $(\mathcal{C}, E) \in \tau_{1}$ and $(\mathcal{D}, E) \in \tau_{2}$.
The complement of soft $\tau_{1} \tau_{2}$-open set is called soft $\tau_{1} \tau_{2}$ closed.

2.15 Definition ([25]) Let $\left(\mathcal{X}, \tau_{1}, \tau_{2}, \mathrm{E}\right)$ be a soft bitopological space and a soft set $(\mathcal{F}, E)$ is called soft $\tau_{1} \tau_{2}$-pre-open set if $(\mathcal{F}, \mathrm{E}) \subseteq \tau_{1} \tau_{2} \operatorname{int}\left(\tau_{1} \tau_{2} \operatorname{cl}(\mathcal{F}, \mathrm{E})\right)$. The complement of soft $\tau_{1} \tau_{2}$-pre-open set is defined to be soft $\tau_{1} \tau_{2}$-pre-closed.

2.16 Definition ([21]) Let $X$ be a nonempty set and $T_{1}, T_{2}$ and $T_{3}$ be a three topologies on $\mathrm{X}$. The set $\mathrm{X}$ together with three topologies is called a tritopological space and is denoted by $\left(X, T_{1}, T_{2}, T_{3}\right)$.

2.17 Definition ([23]) Let $\left(X, T_{1}, T_{2}, T_{3}\right)$ be a tritopological space, a subset $A$ of $\mathrm{X}$ is said to be $\delta^{*}$-open set iff $A \subseteq$ $T_{1} \operatorname{int}\left(T_{2} \operatorname{cl}\left(T_{3} \operatorname{int}(A)\right)\right)$, and the family of all $\delta^{*}$-open sets is denoted by $\delta^{*} .0(X)$. The complement of $\delta^{*}$-open set is called a $\delta^{*}$-closed set

\section{SOFT TRITOPOLOGICAL SPACES}

In this section study of Soft Tritopological spaces is initiated.

3.1 Definition. Let $\left(\mathcal{X}, \tau_{1}, E\right),\left(\mathcal{X}, \tau_{2}, E\right)$ and $\left(X, \tau_{3}, E\right)$ be the three soft topological spaces on $\mathrm{X}$. Then $\left(\mathcal{X}, \tau_{1}, \tau_{2}, \tau_{3}, E\right)$ is called a soft tritopological space.

The three soft topological spaces $\left(\mathcal{X}, \tau_{1}, E\right),\left(\mathcal{X}, \tau_{2}, E\right)$ and $\left(X, \tau_{3}, E\right)$ are independently satisfy the axioms of soft topological space.

The members of $\tau_{1}$ are called $\tau_{1}$ soft open sets and the complements of $\tau_{1}$ soft open sets are called $\tau_{1}$ soft closed sets. And the members of $\tau_{2}$ are called $\tau_{2}$ soft open sets and the complements of $\tau_{2}$ soft open sets are called $\tau_{2}$ soft closed sets. Similarly, the members of $\tau_{3}$ are called $\tau_{3}$ soft open sets and the complements of $\tau_{2}$ soft open sets are called $\tau_{3}$ soft closed sets. Throughout this paper $\left(X, \tau_{1}, \tau_{2}, \tau_{3}, E\right)$ denote soft tritopological space over $\mathcal{X}$.

3.2 Proposition. Let $\left(\mathcal{X}, \tau_{1}, \tau_{2}, \tau_{3}, E\right)$ be a soft tritopological space over $\mathcal{X}$. We define:

$$
\begin{aligned}
\tau_{1 \mathrm{e}} & =\left\{\mathcal{F}(\mathrm{e}):(\mathcal{F}, \mathrm{E}) \in \tau_{1}\right\} \\
\tau_{2 \mathrm{e}} & =\left\{\mathrm{G}(\mathrm{e}):(\mathrm{G}, \mathrm{E}) \in \tau_{2}\right\} \\
\tau_{3 \mathrm{e}} & =\left\{\mathcal{H}(\mathrm{e}):(\mathcal{H}, \mathrm{E}) \in \tau_{3}\right\}
\end{aligned}
$$

for each e $\in \mathrm{E}$. Then $\left(X, \tau_{1 \mathrm{e}}, \tau_{2 \mathrm{e}}, \tau_{3 \mathrm{e}}\right)$ is a tritopological space.

Proof: Follows from the fact that $\tau_{1 \mathrm{e}}, \tau_{2 \mathrm{e}}$ and $\tau_{3 \mathrm{e}}$ are topologies on $X$ for each e $\in \mathrm{E}$.

3.3 Example. Let $\mathrm{X}=\left\{x_{1}, x_{2}, x_{3}\right\}, \quad \mathrm{E}=\left\{e_{1}, e_{2}\right\}$,

$\tau_{1}=\left\{\Phi, \mathcal{X},\left(\mathcal{F}_{1}, \mathrm{E}\right),\left(\mathcal{F}_{2}, \mathrm{E}\right),\left(\mathcal{F}_{3}, \mathrm{E}\right),\left(\mathcal{F}_{4}, \mathrm{E}\right)\right\}$,

$\tau_{2}=\left\{\Phi, \mathcal{X},(\mathcal{X}, \mathrm{E}),\left(\mathrm{G}_{1}, \mathrm{E}\right),\left(\mathrm{G}_{2}, \mathrm{E}\right),\left(\mathrm{G}_{3}, \mathrm{E}\right),\left(\mathrm{G}_{4}, \mathrm{E}\right),\left(\mathrm{G}_{5}, \mathrm{E}\right)\right\}$ and $\tau_{3}=\left\{\Phi, \mathcal{X},\left(\mathcal{H}_{1}, \mathrm{E}\right),\left(\mathcal{H}_{2}, \mathrm{E}\right),\left(\mathcal{H}_{3}, \mathrm{E}\right),\left(\mathcal{H}_{4}, \mathrm{E}\right),\left(\mathcal{H}_{5}, \mathrm{E}\right)\right.$, $\left.\left(\mathcal{H}_{6}, \mathrm{E}\right),\left(\mathcal{H}_{7}, \mathrm{E}\right)\right\}$,

Where $\left(\mathcal{F}_{1}, \mathrm{E}\right),\left(\mathcal{F}_{2}, \mathrm{E}\right),\left(\mathcal{F}_{3}, \mathrm{E}\right),\left(\mathcal{F}_{4}, \mathrm{E}\right),\left(\mathcal{F}_{5}, \mathrm{E}\right)$, $\left(G_{1}, E\right),\left(G_{2}, E\right),\left(G_{3}, E\right),\left(G_{4}, E\right),\left(G_{5}, E\right)$

$\left(\mathcal{H}_{1}, \mathrm{E}\right),\left(\mathcal{H}_{2}, \mathrm{E}\right),\left(\mathcal{H}_{3}, \mathrm{E}\right),\left(\mathcal{H}_{4}, \mathrm{E}\right)$ are soft open sets over $\mathcal{X}$. Where defined as follows:

$$
\begin{aligned}
& \mathcal{F}_{1}\left(e_{1}\right)=\left\{x_{2}\right\} \quad, \quad \mathcal{F}_{1}\left(e_{2}\right)=\left\{x_{1}\right\} \\
& \mathcal{F}_{2}\left(e_{1}\right)=\left\{x_{2}, x_{3}\right\} \quad, \quad \mathcal{F}_{2}\left(e_{2}\right)=\left\{x_{1}, x_{2}\right\} \\
& \mathcal{F}_{3}\left(e_{1}\right)=\left\{x_{1}, x_{2}\right\} \quad, \quad \mathcal{F}_{3}\left(e_{2}\right)=\mathrm{X} \\
& \mathcal{F}_{4}\left(e_{1}\right)=\left\{x_{1}, x_{2}\right\} \quad, \quad \mathcal{F}_{4}\left(e_{2}\right)=\left\{x_{1}, x_{3}\right\} \\
& \mathcal{F}_{5}\left(e_{1}\right)=\left\{x_{2}\right\} \quad, \quad \mathcal{F}_{5}\left(e_{2}\right)=\left\{x_{1}, x_{2}\right\} \\
& \mathrm{G}_{1}\left(e_{1}\right)=\left\{x_{1}, x_{3}\right\} \quad, \quad \mathrm{G}_{1}\left(e_{2}\right)=\left\{x_{1}, x_{3}\right\} \\
& \mathrm{G}_{2}\left(e_{1}\right)=\left\{x_{1}\right\} \quad, \quad \mathrm{G}_{2}\left(e_{2}\right)=\left\{x_{2}, x_{3}\right\} \\
& \mathrm{G}_{3}\left(e_{1}\right)=\left\{x_{1}, x_{2}\right\} \quad, \quad \mathrm{G}_{3}\left(e_{2}\right)=\left\{x_{3}\right\}
\end{aligned}
$$




$$
\begin{array}{lll}
\mathrm{G}_{4}\left(e_{1}\right)=\left\{x_{1}\right\} & , & \mathrm{G}_{4}\left(e_{2}\right)=\left\{x_{3}\right\} \\
\mathrm{G}_{5}\left(e_{1}\right)=\left\{x_{1}, x_{3}\right\} & , & \\
& \mathrm{G}_{5}\left(e_{2}\right)=\mathrm{X} \\
\mathcal{H}_{1}\left(e_{1}\right)=\left\{x_{1}\right\} & , & \mathcal{H}_{1}\left(e_{2}\right)=\left\{x_{2}\right\} \\
\mathcal{H}_{2}\left(e_{1}\right)=\left\{x_{1}, x_{2}\right\} & , & \mathcal{H}_{2}\left(e_{2}\right)=\left\{x_{2}\right\} \\
\mathcal{H}_{3}\left(e_{1}\right)=\left\{x_{2}\right\} & , & \mathcal{H}_{3}\left(e_{2}\right)=\left\{x_{2}\right\} \\
\mathcal{H}_{4}\left(e_{1}\right)=\emptyset & , & \mathcal{H}_{4}\left(e_{2}\right)=\left\{x_{2}\right\}
\end{array}
$$

Then $\left(\mathcal{X}, \tau_{1}, \tau_{2}, \tau_{3}, E\right)$ is a soft tritopological space. Notice;

$\tau_{1_{e_{1}}}=\left\{\emptyset, \mathrm{X},\left\{x_{1}\right\},\left\{x_{1}, x_{2}\right\},\left\{x_{1}, x_{3}\right\}\right\}$,

$\tau_{1_{e_{2}}}=\left\{\emptyset, \mathrm{X},\left\{x_{3}\right\},\left\{x_{1}, x_{3}\right\},\left\{x_{2}, x_{3}\right\}\right\}$,

$\tau_{2_{e_{1}}}=\left\{\emptyset, \mathrm{X},\left\{x_{1}\right\},\left\{x_{1}, x_{2}\right\},\left\{x_{1}, x_{3}\right\}\right\}$,

$\tau_{2_{e_{2}}}=\left\{\emptyset, \mathrm{X},\left\{x_{3}\right\},\left\{x_{1}, x_{3}\right\},\left\{x_{2}, x_{3}\right\}\right\}$ and

$\tau_{3_{e_{1}}}=\left\{\emptyset, \mathrm{X},\left\{x_{2}\right\},\left\{x_{3}\right\},\left\{x_{1}, x_{2}\right\},\left\{x_{2}, x_{3}\right\}\right\}$,

$\tau_{3_{e_{2}}}=\left\{\emptyset, X,\left\{x_{1}\right\},\left\{x_{1}, x_{2}\right\},\left\{x_{1}, x_{3}\right\}\right\}$

Are topologies on $\mathrm{X}$.

3.4 Example. In the scientific experiments in cellular biology which can be applied to detect whether a concentrations of plant extract are used as a therapy of the certain disease or not.

Let our universal set contains three concentrations of the certain plant extract, i.e. $\mathrm{X}=\left\{x_{1}, x_{2}, x_{3}\right\}$.

There are two categories of experiments: in vitro studies and in vivo studies. In vitro experiments are conducted outside of organisms or cells, i.e., in a laboratory environment using test tubes, petri dishes, etc. in vivo experiments are conducted inside of organisms or cells, then the set of parameters $\mathrm{E}=\{$ in vitro, in vivo $\}$, after the experiments was conducted, the experiment tested for three stages (three time periods), the first period is after 1 month, the second period is after 3 months and the third period is after 6 months. The three time periods represented as three soft topological spaces $\tau_{1}, \tau_{2}$ and $\tau_{3}$.

When testing the experiment after 1 month, we obtain the soft open set $(\mathcal{F}, E)$ over $\mathcal{X}$, where defined as:

$$
\mathcal{F}(\text { in vitro })=\emptyset \quad, \quad \mathcal{F}(\text { in vivo })=\left\{x_{2}\right\}
$$

i.e. there is no visible effect for all concentrations of plant extract on the ( in vitro) study. But the effect of the second concentration of plant extract $\left(x_{2}\right)$ is the best one in the (in vivo) study.

When testing the experiment after 3 months, we obtain the soft open set $(\mathrm{G}, \mathrm{E})$ over $\mathcal{X}$, where defined as:

$$
\mathrm{G}(\text { in vitro })=\left\{x_{2}\right\} \quad, \quad \mathrm{G}(\text { in vivo })=\left\{x_{2}, x_{3}\right\}
$$

i.e. the best effect in the ( in vitro) study is for the concentration $x_{2}$. But the best effect in the (in vivo) study are for $x_{2}$ and $x_{3}$ the second and third concentrations of plant extract.

And when testing the experiment after 6 months, we obtain the soft open set $(\mathcal{H}, \mathrm{E})$ over $\mathcal{X}$, where defined as:

$\mathcal{H}($ in vitro $)=\left\{x_{1}, x_{3}\right\} \quad, \quad \mathcal{H}($ in vivo $)=\left\{x_{1}\right\}$

i.e. the best effect is for $x_{1}$ and $x_{3}$ concentrations in the (in vitro) study. But the $x_{1}$ concentration has the best effect in the (in vivo) study.

Then $\left(\mathcal{X}, \tau_{1}, \tau_{2}, \tau_{3}, E\right)$ is a soft tritopological space.

This is only a simple example to show the possibility of using this concept for natural applications.
3.5 Theorem. If $\left(X, \tau_{1}, \tau_{2}, \tau_{3}, E\right)$ is a soft tritopological space then $\tau=\tau_{1} \cap \tau_{2} \cap \tau_{3}$ is a soft topological space over $\mathcal{X}$. Proof:

1) $\Phi, \mathcal{X}$ belong to $\tau_{1} \cap \tau_{2} \cap \tau_{3}=\tau$.

2) Let $\left\{\left(\mathcal{F}_{i}, E\right): i \in I\right\}$ be a family of soft sets in $\tau_{1} \cap \tau_{2} \cap \tau_{3}=\tau$.

Then $\left\{\left(\mathcal{F}_{i}, E\right) \in \tau_{1}\right\},\left\{\left(\mathcal{F}_{i}, E\right) \in \tau_{2}\right\}$ and $\left\{\left(\mathcal{F}_{i}, E\right) \in \tau_{3}\right\}$ for all $i \in I$,

therefore $\bigcup_{i \in I}\left(\mathcal{F}_{i}, E\right) \in \tau_{1} \quad, \quad \bigcup_{i \in I}\left(\mathcal{F}_{i}, E\right) \in \tau_{2} \quad$ and $\bigcup_{i \in I}\left(\mathcal{F}_{i}, E\right) \in \tau_{3}$. Thus $\bigcup_{i \in I}\left(\mathcal{F}_{i}, E\right) \in \tau_{1} \cap \tau_{2} \cap \tau_{3}=\tau$. 3) Let $(\mathcal{F}, E),(G, E) \in \tau_{1} \cap \tau_{2} \cap \tau_{3}=\tau$. Then $(\mathcal{F}, E)$, $(\mathrm{G}, E) \in \tau_{1},(\mathcal{F}, E),(\mathrm{G}, E) \in \tau_{2} \quad$ and $(\mathcal{F}, E),(\mathrm{G}, E) \in \tau_{3}$. Since $(\mathcal{F}, E) \cap(\mathrm{G}, E) \in \tau_{1},(\mathcal{F}, E) \cap(\mathrm{G}, E) \in \tau_{2}$ and $(\mathcal{F}, E) \cap(\mathrm{G}, E) \in \tau_{3}$.

Therefore $(\mathcal{F}, E) \cap(\mathrm{G}, E) \in \tau_{1} \cap \tau_{2} \cap \tau_{3}=\tau$.

Thus $\tau_{1} \cap \tau_{2} \cap \tau_{3}=\tau$ defines a soft topology on $\mathcal{X}$.

3.6 Remark. If $\left(\mathcal{X}, \tau_{1}, \tau_{2}, \tau_{3}, E\right)$ is a soft tritopological space then $\tau_{1} \cup \tau_{2} \cup \tau_{3}$ is not a soft topological space over $\mathcal{X}$.

3.7 Example. Let $\mathrm{X}=\left\{x_{1}, x_{2}, x_{3}\right\}, \mathrm{E}=\left\{e_{1}, e_{2}\right\}$, $\tau_{1}=\left\{\Phi, \mathcal{X},\left(\mathcal{F}_{1}, \mathrm{E}\right),\left(\mathcal{F}_{2}, \mathrm{E}\right),\left(\mathcal{F}_{3}, \mathrm{E}\right),\left(\mathcal{F}_{4}, \mathrm{E}\right),\left(\mathcal{F}_{5}, \mathrm{E}\right)\right\}$, $\tau_{2}=\left\{\Phi, \mathcal{X},\left(\mathrm{G}_{1}, \mathrm{E}\right),\left(\mathrm{G}_{2}, \mathrm{E}\right),\left(\mathrm{G}_{3}, \mathrm{E}\right),\left(\mathrm{G}_{4}, \mathrm{E}\right),\left(\mathrm{G}_{5}, \mathrm{E}\right)\right\}$ and $\tau_{3}=\left\{\Phi, \mathcal{X},\left(\mathcal{H}_{1}, \mathrm{E}\right),\left(\mathcal{H}_{2}, \mathrm{E}\right),\left(\mathcal{H}_{3}, \mathrm{E}\right),\left(\mathcal{H}_{4}, \mathrm{E}\right),\left(\mathcal{H}_{5}, \mathrm{E}\right)\right.$, $\left.\left(\mathcal{H}_{6}, \mathrm{E}\right),\left(\mathcal{H}_{7}, \mathrm{E}\right)\right\}$, Where

$\left(\mathcal{F}_{1}, \mathrm{E}\right),\left(\mathcal{F}_{2}, \mathrm{E}\right),\left(\mathcal{F}_{3}, \mathrm{E}\right),\left(\mathcal{F}_{4}, \mathrm{E}\right),\left(\mathcal{F}_{5}, \mathrm{E}\right)$

$\left(G_{1}, E\right),\left(G_{2}, E\right),\left(G_{3}, E\right),\left(G_{4}, E\right),\left(G_{5}, E\right)$

$\left(\mathcal{H}_{1}, \mathrm{E}\right),\left(\mathcal{H}_{2}, \mathrm{E}\right),\left(\mathcal{H}_{3}, \mathrm{E}\right),\left(\mathcal{H}_{4}, \mathrm{E}\right),\left(\mathcal{H}_{5}, \mathrm{E}\right),\left(\mathcal{H}_{6}, \mathrm{E}\right),\left(\mathcal{H}_{7}, \mathrm{E}\right)$ are soft open sets over $\mathcal{X}$. Where defined as follows;

$$
\begin{aligned}
& \mathcal{F}_{1}\left(e_{1}\right)=\left\{x_{2}\right\} \quad, \quad \mathcal{F}_{1}\left(e_{2}\right)=\left\{x_{1}\right\} \\
& \mathcal{F}_{2}\left(e_{1}\right)=\left\{x_{2}, x_{3}\right\} \quad, \quad \mathcal{F}_{2}\left(e_{2}\right)=\left\{x_{1}, x_{2}\right\} \\
& \mathcal{F}_{3}\left(e_{1}\right)=\left\{x_{1}, x_{2}\right\} \quad, \quad \mathcal{F}_{3}\left(e_{2}\right)=\left\{x_{1}, x_{3}\right\} \\
& \mathcal{F}_{4}\left(e_{1}\right)=\left\{x_{2}\right\} \quad, \quad \mathcal{F}_{4}\left(e_{2}\right)=\left\{x_{1}, x_{3}\right\} \\
& \mathcal{F}_{5}\left(e_{1}\right)=\left\{x_{2}\right\} \quad, \quad \mathcal{F}_{5}\left(e_{2}\right)=\emptyset \\
& \mathrm{G}_{1}\left(e_{1}\right)=\mathrm{X} \\
& \mathrm{G}_{2}\left(e_{1}\right)=\left\{x_{1}\right\} \\
& \mathrm{G}_{3}\left(e_{1}\right)=\left\{x_{1}, x_{3}\right\} \\
& \mathrm{G}_{4}\left(e_{1}\right)=\left\{x_{1}, x_{2}\right\} \\
& \mathrm{G}_{5}\left(e_{1}\right)=\emptyset \\
& \mathrm{G}_{1}\left(e_{2}\right)=\left\{x_{1}, x_{3}\right\} \\
& \mathrm{G}_{2}\left(e_{2}\right)=\left\{x_{2}, x_{3}\right\} \\
& \mathrm{G}_{3}\left(e_{2}\right)=\mathrm{X} \\
& \mathrm{G}_{4}\left(e_{2}\right)=\left\{x_{3}\right\} \\
& \mathrm{G}_{5}\left(e_{2}\right)=\left\{x_{3}\right\} \\
& \mathcal{H}_{1}\left(e_{1}\right)=\left\{x_{1}, x_{2}\right\} \\
& \mathcal{H}_{1}\left(e_{2}\right)=\left\{x_{1}, x_{2}\right\} \\
& \mathcal{H}_{2}\left(e_{1}\right)=\left\{x_{2}\right\} \\
& \mathcal{H}_{3}\left(e_{1}\right)=\left\{x_{2}, x_{3}\right\} \\
& \mathcal{H}_{4}\left(e_{1}\right)=\left\{x_{2}\right\} \\
& \mathcal{H}_{5}\left(e_{1}\right)=\left\{x_{1}, x_{2}\right\} \\
& \mathcal{H}_{6}\left(e_{1}\right)=\mathrm{X} \\
& \mathcal{H}_{7}\left(e_{1}\right)=\left\{x_{2}, x_{3}\right\} \\
& \mathcal{H}_{2}\left(e_{2}\right)=\left\{x_{1}, x_{3}\right\} \\
& \mathcal{H}_{3}\left(e_{2}\right)=\left\{x_{1}\right\} \\
& \mathcal{H}_{4}\left(e_{2}\right)=\left\{x_{1}\right\} \\
& \mathcal{H}_{5}\left(e_{2}\right)=\mathrm{X} \\
& \mathcal{H}_{6}\left(e_{2}\right)=\left\{x_{1}, x_{2}\right\} \\
& \mathcal{H}_{7}\left(e_{2}\right)=\left\{x_{1}, x_{3}\right\}
\end{aligned}
$$

Then $\left(\mathcal{X}, \tau_{1}, \tau_{2}, \tau_{3}, E\right)$ is a soft tritopological space. Now we define: $\tau=\tau_{1} \cup \tau_{2} \cup \tau_{3}=\left\{\Phi, \mathcal{X},\left(\mathcal{F}_{1}, \mathrm{E}\right),\left(\mathcal{F}_{2}, \mathrm{E}\right),\left(\mathcal{F}_{3}, \mathrm{E}\right)\right.$, $\left(\mathcal{F}_{4}, \mathrm{E}\right),\left(\mathcal{F}_{5}, \mathrm{E}\right),\left(\mathrm{G}_{1}, \mathrm{E}\right),\left(\mathrm{G}_{2}, \mathrm{E}\right),\left(\mathrm{G}_{3}, \mathrm{E}\right),\left(\mathrm{G}_{4}, \mathrm{E}\right),\left(\mathrm{G}_{5}, \mathrm{E}\right)$, $\left.\left(\mathcal{H}_{1}, \mathrm{E}\right),\left(\mathcal{H}_{2}, \mathrm{E}\right),\left(\mathcal{H}_{3}, \mathrm{E}\right),\left(\mathcal{H}_{4}, \mathrm{E}\right),\left(\mathcal{H}_{5}, \mathrm{E}\right),\left(\mathcal{H}_{6}, \mathrm{E}\right),\left(\mathcal{H}_{7}, \mathrm{E}\right)\right\}$ If we take $\left(\mathcal{F}_{5}, \mathrm{E}\right) \cup\left(\mathrm{G}_{5}, \mathrm{E}\right) \cup\left(\mathcal{H}_{5}, \mathrm{E}\right)=(\mathcal{D}, \mathrm{E})$, then

$$
\begin{aligned}
\mathcal{D}\left(e_{1}\right) & =\mathcal{F}_{5}\left(e_{1}\right) \cup \mathrm{G}_{5}\left(e_{1}\right) \cup \mathcal{H}_{5}\left(e_{1}\right) \\
& =\left\{x_{2}, x_{3}\right\} \cup \emptyset \cup\left\{x_{2}\right\}=\left\{x_{2}, x_{3}\right\} \quad \text {, and } \\
\mathcal{D}\left(e_{2}\right) & =\mathcal{F}_{5}\left(e_{2}\right) \cup G_{5}\left(e_{2}\right) \cup \mathcal{H}_{5}\left(e_{2}\right) \\
& =\emptyset \cup\left\{x_{3}\right\} \cup\left\{x_{1}, x_{2}\right\}=X
\end{aligned}
$$

but $(\mathcal{D}, \mathrm{E}) \notin \tau$. Thus $\tau$ is not a soft topology on $X$. 


\section{SOME KINDS OF SOFT OPEN SETS IN SOFT TRITOPOLOGICAL SPACES}

4.1 Definition. Let $\left(\mathcal{X}, \tau_{1}, \tau_{2}, \tau_{3}, E\right)$ be a soft tritopological space, and $(\mathcal{F}, E)$ is soft set in $\mathcal{X}$, then:

(i) $(\mathcal{F}, E)$ is called a soft $\tau_{1} \tau_{2} \tau_{3}$-open set if $(\mathcal{F}, E)=$ $(\mathcal{B}, E) \cup(\mathcal{C}, E) \cup(\mathcal{D}, E)$, where $(\mathcal{B}, E) \in \tau_{1},(\mathcal{C}, E) \in \tau_{2}$ and $(\mathcal{D}, E) \in \tau_{3}$. The complement of soft $\tau_{1} \tau_{2} \tau_{3}$-open set is called soft $\tau_{1} \tau_{2} \tau_{3}$-closed. The family of all soft $\tau_{1} \tau_{2} \tau_{3}$-open sets is denoted by $S . \tau_{1} \tau_{2} \tau_{3} . O(X)$. And the family of all soft $\tau_{1} \tau_{2} \tau_{3}$-closed sets is denoted by $S . \tau_{1} \tau_{2} \tau_{3} . \mathrm{C}(X)$.

(ii) $(\mathcal{F}, E)$ is called a soft $\tau_{1} \tau_{2} \tau_{3}$-pre-open set if $(\mathcal{F}, E) \subseteq$ s. $\tau_{1} \tau_{2} \tau_{3} \operatorname{int}\left(\mathrm{s} . \tau_{1} \tau_{2} \tau_{3} \operatorname{cl}(\mathcal{F}, E)\right)$. The complement of soft $\tau_{1} \tau_{2} \tau_{3}$-pre-open set is defined to be soft $\tau_{1} \tau_{2} \tau_{3}$-pre-closed.

(iii) $(\mathcal{F}, E)$ is called a soft $\tau_{1} \tau_{2} \tau_{3}$ - $\alpha$-open set(or soft tri- $\alpha$-open set) if $(\mathcal{F}, \mathrm{E}) \subseteq$ s. $\tau_{1} \tau_{2} \tau_{3} \operatorname{int}\left(\mathrm{s} . \tau_{1} \tau_{2} \tau_{3} \operatorname{cl}\left(\mathrm{s} . \tau_{1} \tau_{2} \tau_{3} \operatorname{int}(\mathcal{F}, \mathrm{E})\right)\right)$. The complement of soft $\tau_{1} \tau_{2} \tau_{3}-\alpha$-open(or soft tri- $\alpha$-open set) set is defined to be soft $\tau_{1} \tau_{2} \tau_{3}-\alpha$-closed(or soft tri- $\alpha$-closed set).

(iv) $(\mathcal{F}, E)$ is called a soft $\delta^{*}$-open set iff $(\mathcal{F}, E) \subseteq$ s. $\tau_{1} \operatorname{int}\left(s . \tau_{2} \operatorname{cl}\left(s . \tau_{3} \operatorname{int}(\mathcal{F}, E)\right)\right)$. The complement of soft $\delta^{*}-$ open set is called a soft $\delta^{*}$-closed set.

4.2 Definition. Let $\left(\mathcal{X}, \tau_{1}, \tau_{2}, \tau_{3}, E\right)$ be a soft tritopological space, and $(\mathcal{F}, E)$ is a soft set in $\mathcal{X}$, then:

(i) The soft $\tau_{1} \tau_{2} \tau_{3}$-closure of $(\mathcal{F}, E)$, denoted by s. $\tau_{1} \tau_{2} \tau_{3} \operatorname{cl}(\mathcal{F}, E)$ is defined by:

s. $\tau_{1} \tau_{2} \tau_{3} \operatorname{cl}(\mathcal{F}, E)=\bigcap\{(\mathcal{C}, E):(\mathcal{F}, E) \subseteq(\mathcal{C}, E)$, and $(\mathcal{C}, E)$ is soft $\tau_{1} \tau_{2} \tau_{3}$-closed $\}$

(ii) The soft $\tau_{1} \tau_{2} \tau_{3}$-interior of $(\mathcal{F}, E)$, denoted by s. $\tau_{1} \tau_{2} \tau_{3} \operatorname{int}(\mathcal{F}, E)$ is defined by:

s. $\tau_{1} \tau_{2} \tau_{3} \operatorname{int}(\mathcal{F}, E)=\bigcup\{(\mathcal{B}, E):(\mathcal{B}, E) \subseteq(\mathcal{F}, E)$, and $(\mathcal{B}, E)$ is soft $\tau_{1} \tau_{2} \tau_{3}$-open\}

4.3 Example. Let $\mathrm{X}=\left\{x_{1}, x_{2}, x_{3}\right\}, \mathrm{E}=\left\{e_{1}, e_{2}\right\}$, $\tau_{1}=\{\Phi, \mathcal{X},(\mathcal{F}, \mathrm{E})\}, \tau_{2}=\left\{\Phi, \mathcal{X},\left(\mathrm{G}_{1}, \mathrm{E}\right),\left(\mathrm{G}_{2}, \mathrm{E}\right)\right\} \quad$ and $\tau_{3}=\left\{\Phi, \mathcal{X},\left(\mathcal{H}_{1}, \mathrm{E}\right),\left(\mathcal{H}_{2}, \mathrm{E}\right),\left(\mathcal{H}_{3}, \mathrm{E}\right)\right\}$ are soft topologies on $\mathcal{X}$. Where $(\mathcal{F}, \mathrm{E}),\left(\mathrm{G}_{1}, \mathrm{E}\right),\left(\mathrm{G}_{2}, \mathrm{E}\right),\left(\mathrm{G}_{3}, \mathrm{E}\right),\left(\mathcal{H}_{1}, \mathrm{E}\right)$, $\left(\mathcal{H}_{2}, \mathrm{E}\right),\left(\mathcal{H}_{3}, \mathrm{E}\right)$ are soft open sets over $\mathcal{X}$, defined as follows;

$$
\begin{array}{lll}
\mathcal{F}\left(e_{1}\right)=\left\{x_{2}\right\} & , & \mathcal{F}\left(e_{2}\right)=\left\{x_{1}, x_{2}\right\} \\
\mathrm{G}_{1}\left(e_{1}\right)=\left\{x_{1}\right\} & , & \mathrm{G}_{1}\left(e_{2}\right)=\left\{x_{1}, x_{3}\right\} \\
\mathrm{G}_{2}\left(e_{1}\right)=\left\{x_{1}, x_{2}\right\} & , & \mathrm{G}_{2}\left(e_{2}\right)=\mathrm{X} \\
\mathrm{G}_{3}\left(e_{1}\right)=\left\{x_{2}\right\} & , & \mathrm{G}_{3}\left(e_{2}\right)=\left\{x_{2}\right\} \\
& & \\
\mathcal{H}_{1}\left(e_{1}\right)=\left\{x_{2}, x_{3}\right\} & & \\
\mathcal{H}_{2}\left(e_{1}\right)=\emptyset & & \mathcal{H}_{1}\left(e_{2}\right)=\left\{x_{1}\right\} \\
\mathcal{H}_{3}\left(e_{1}\right)=\left\{x_{2}, x_{3}\right\} & , & \mathcal{H}_{2}\left(e_{2}\right)=\left\{x_{2}\right\} \\
& & \mathcal{H}_{3}\left(e_{2}\right)=\left\{x_{1}, x_{2}\right\}
\end{array}
$$

Then $\left(X, \tau_{1}, \tau_{2}, \tau_{3}, E\right)$ is a soft tritopological space. And all the soft $\tau_{1} \tau_{2} \tau_{3}$-open sets are in the family: $S . \tau_{1} \tau_{2} \tau_{3} . \mathrm{O}(\mathcal{X})=$ $\left\{\Phi, \mathcal{X},(\mathcal{F}, \mathrm{E}),\left(\mathrm{G}_{1}, \mathrm{E}\right),\left(\mathrm{G}_{2}, \mathrm{E}\right),\left(\mathrm{G}_{3}, \mathrm{E}\right),\left(\mathcal{H}_{1}, \mathrm{E}\right),\left(\mathcal{H}_{2}, \mathrm{E}\right),\left(\mathcal{H}_{3}, \mathrm{E}\right)\right\} \cup$ $\left\{\left(\mathrm{G}_{1}, \mathrm{E}\right) \cup\left(\mathcal{H}_{1}, \mathrm{E}\right),\left(\mathrm{G}_{1}, \mathrm{E}\right) \cup\left(\mathcal{H}_{2}, \mathrm{E}\right)\right\}$

$=\tau_{1} \cup \tau_{2} \cup \tau_{3} \cup\left\{\left(\mathrm{G}_{1}, \mathrm{E}\right) \cup\left(\mathcal{H}_{1}, \mathrm{E}\right),\left(\mathrm{G}_{1}, \mathrm{E}\right) \cup\left(\mathcal{H}_{2}, \mathrm{E}\right)\right\}$

Now, we find the soft $\tau_{1} \tau_{2} \tau_{3}$-closed sets: $S . \tau_{1} \tau_{2} \tau_{3}$. C $(\mathcal{X})=$ $\left\{\Phi, \mathcal{X},(\mathcal{F}, \mathrm{E})^{c},\left(\mathrm{G}_{1}, \mathrm{E}\right)^{c},\left(\mathrm{G}_{2}, \mathrm{E}\right)^{c},\left(\mathrm{G}_{3}, \mathrm{E}\right)^{c},\left(\mathcal{H}_{1}, \mathrm{E}\right)^{c},\left(\mathcal{H}_{2}, \mathrm{E}\right)^{c},\left(\mathcal{H}_{3}, \mathrm{E}\right)^{c}\right\} \cup$ $\left\{\left[\left(\mathrm{G}_{1}, \mathrm{E}\right) \cup\left(\mathcal{H}_{1}, \mathrm{E}\right)\right]^{c},\left[\left(\mathrm{G}_{1}, \mathrm{E}\right) \cup\left(\mathcal{H}_{2}, \mathrm{E}\right)\right]^{c}\right\}$

Where defined as follows;

$$
\begin{array}{lll}
F^{c}\left(e_{1}\right)=\left\{x_{1}, x_{3}\right\} & , & F^{c}\left(e_{2}\right)=\left\{x_{3}\right\} \\
\mathrm{G}_{1}{ }^{c}\left(e_{1}\right)=\left\{x_{2}, x_{3}\right\} & , & \mathrm{G}_{1}{ }^{c}\left(e_{2}\right)=\left\{x_{2}\right\} \\
\mathrm{G}_{2}{ }^{c}\left(e_{1}\right)=\left\{x_{3}\right\} & , & \mathrm{G}_{2}{ }^{c}\left(e_{2}\right)=\emptyset \\
\mathrm{G}_{3}{ }^{c}\left(e_{1}\right)=\left\{x_{1}, x_{3}\right\} & , & \mathrm{G}_{3}{ }^{c}\left(e_{2}\right)=\left\{x_{1}, x_{3}\right\} \\
& & \\
\mathcal{H}_{1}{ }^{c}\left(e_{1}\right)=\left\{x_{1}\right\} & , & \mathcal{H}_{1}{ }^{c}\left(e_{2}\right)=\left\{x_{2}, x_{3}\right\} \\
\mathcal{H}_{2}{ }^{c}\left(e_{1}\right)=X & , & \mathcal{H}_{2}{ }^{c}\left(e_{2}\right)=\left\{x_{1}, x_{3}\right\} \\
\mathcal{H}_{3}{ }^{c}\left(e_{1}\right)=\left\{x_{1}\right\} & , & \mathcal{H}_{3}{ }^{c}\left(e_{2}\right)=\left\{x_{3}\right\} \\
& & \\
{\left[\left(\mathrm{G}_{1}, \mathrm{E}\right) \cup\left(\mathcal{H}_{1}, \mathrm{E}\right)\right]^{c}\left(e_{1}\right)=[X]^{c}=\emptyset,} \\
{\left[\left(\mathrm{G}_{1}, \mathrm{E}\right) \cup\left(\mathcal{H}_{1}, \mathrm{E}\right)\right]^{c}\left(e_{2}\right)=\left[\left\{x_{1}, x_{3}\right\}\right]^{c}=\left\{x_{2}\right\}} \\
{\left[\left(\mathrm{G}_{1}, \mathrm{E}\right) \cup\left(\mathcal{H}_{2}, \mathrm{E}\right)\right]^{c}\left(e_{1}\right)=\left[\left\{x_{1}\right\}\right]^{c}=\left\{x_{2}, x_{3}\right\},} \\
{\left[\left(\mathrm{G}_{1}, \mathrm{E}\right) \cup\left(\mathcal{H}_{2}, \mathrm{E}\right)\right]^{c}\left(e_{2}\right)=[X]^{c}=\emptyset}
\end{array}
$$

If we take the soft set $(\mathcal{A}, \mathrm{E})$ which defined as: $\mathcal{A}\left(e_{1}\right)=\left\{x_{2}\right\} \quad, \quad \mathcal{A}\left(e_{2}\right)=\left\{x_{3}\right\}$

Then, s. $\tau_{1} \tau_{2} \tau_{3} \operatorname{int}\left(\right.$ s. $\left.\tau_{1} \tau_{2} \tau_{3} \operatorname{cl}(\mathcal{A}, \mathrm{E})\right)=\mathrm{s} . \tau_{1} \tau_{2} \tau_{3} \operatorname{int}\left(\left(\mathcal{H}_{2}, \mathrm{E}\right)^{c}\right)$ $=\left\{\left(\mathrm{G}_{1}, \mathrm{E}\right) \cup\left(\mathcal{H}_{1}, \mathrm{E}\right)\right\}$,

$(\mathcal{A}, \mathrm{E}) \subseteq\left\{\left(\mathrm{G}_{1}, \mathrm{E}\right) \cup\left(\mathcal{H}_{1}, \mathrm{E}\right)\right\}$

Hence $(\mathcal{A}, \mathrm{E})$ is a soft $\tau_{1} \tau_{2} \tau_{3}$-pre-open set.

If we take the soft set $(\mathcal{A}, \mathrm{E})$ which defined as:

$$
\mathcal{A}\left(e_{1}\right)=\left\{x_{1}\right\}, \mathcal{A}\left(e_{2}\right)=\left\{x_{1}, x_{3}\right\} \text { then: }
$$

s. $\tau_{1} \tau_{2} \tau_{3} \operatorname{int}\left(\mathrm{s} . \tau_{1} \tau_{2} \tau_{3} \operatorname{cl}(\mathcal{A}, \mathrm{E})\right)=$ s. $\tau_{1} \tau_{2} \tau_{3} \operatorname{int}\left(\left(\mathrm{G}_{3}, \mathrm{E}\right)^{c}\right)=$ $\left(\mathrm{G}_{1}, \mathrm{E}\right)$. Hence $(\mathcal{A}, \mathrm{E}) \subseteq\left(\mathrm{G}_{1}, \mathrm{E}\right)$.

Therefore the soft set $(\mathcal{A}, \mathrm{E})$ is a soft $\tau_{1} \tau_{2} \tau_{3}$-pre-open set.

To find soft $\tau_{1} \tau_{2} \tau_{3}-\alpha$-open sets (or soft tri- $\alpha$-open sets), we take the soft set $(\mathcal{A}, \mathrm{E})$ which defined as:

$\mathcal{A}\left(e_{1}\right)=\left\{x_{2}, x_{3}\right\}, \mathcal{A}\left(e_{2}\right)=\left\{x_{1}, x_{2}\right\}$

then: $\quad$ s. $\tau_{1} \tau_{2} \tau_{3} \operatorname{int}\left(\right.$ s. $\tau_{1} \tau_{2} \tau_{3} \operatorname{cl}\left(\right.$ s. $\left.\left.\tau_{1} \tau_{2} \tau_{3} \operatorname{int}(\mathcal{A}, \mathrm{E})\right)\right)=$ s. $\tau_{1} \tau_{2} \tau_{3} \operatorname{int}\left(\mathrm{s} . \tau_{1} \tau_{2} \tau_{3} \operatorname{cl}\left(\mathcal{H}_{3}, \mathrm{E}\right)\right)=\mathrm{s} . \tau_{1} \tau_{2} \tau_{3} \operatorname{int}((\mathcal{X}, \mathrm{E}))=(\mathcal{X}, \mathrm{E})$ Hence $(\mathcal{A}, \mathrm{E}) \subseteq(\mathcal{X}, \mathrm{E})$

Therefore the soft set $(\mathcal{A}, \mathrm{E})$ is a $\tau_{1} \tau_{2} \tau_{3}-\alpha$-open sets (or soft tri- $\alpha$-open sets)

Now, if we take the soft subset $(\mathcal{A}, \mathrm{E})$ of $(\mathcal{X}, \mathrm{E})$ which defined as: $\mathcal{A}\left(e_{1}\right)=\left\{x_{2}, x_{3}\right\}, \mathcal{A}\left(e_{2}\right)=\left\{x_{1}\right\}$ then:

s. $\tau_{1} \operatorname{int}\left(\right.$ s. $\tau_{2} \operatorname{cl}\left(\right.$ s. $\left.\left.\tau_{3} \operatorname{int}(\mathcal{A}, \mathrm{E})\right)\right)=$ s. $\tau_{1} \operatorname{int}\left(\right.$ s. $\left.\tau_{2} \operatorname{cl}\left(\left(\mathcal{H}_{1}, \mathrm{E}\right)\right)\right)=$ s. $\tau_{1} \operatorname{int}((X, \mathrm{E}))=(\mathcal{X}, \mathrm{E})$

Hence $(\mathcal{A}, \mathrm{E}) \subseteq(\mathcal{X}, \mathrm{E})$.

Therefore the soft set $(\mathcal{A}, \mathrm{E})$ is a soft $\delta^{*}$-open set.

\section{CONCLUSION}

The concept of soft topological spaces is generalized to soft tritopological spaces. Some basic notions of classical and generalized concepts have been studied. the purpose of this paper is just to initiate the concept, and there is a lot of scope for the researchers to make their investigations in this field, i.e. this is a beginning of some new generalized structures and the concepts like separation axioms and another basic concepts can be studied. This subject can be extended in the future Research with some natural applications. Also can be study the relationships among some types of soft open sets in soft tritopological spaces.

\section{REFERENCES}

[1] D. Molodtsov, Soft set theory first results, Comput. Math. Appl. 37 (1999) 19-31.

[2] P.K. Maji, A. R. Roy, R. Biswas, An application of soft sets in a decision making problem, Comput. Math. Appl. 44 (2002) 1077-1083. 
[3] D. Pie, D. Miao, From soft sets to information systems, Granu. comput. IEEE Inter. Conf. 2 (2005) 617-621.

[4] M.I. Ali, F. Feng, X.Y. Liu, W.K. Min, M. Shabir, On some new operations in soft set theory, Comput. Math. Appl. 57 (2009) 1547-1553.

[5] M. I. Ali, M. Shabir, M. Naz, Algebraic structures of soft sets associated with new operations, Comput. Math. Appl. 61 (2011) 2647-2654.

[6] P.K. Maji, R. Biswas, A.R. Roy, Soft set theory, Comput. Math. Appl. 45 (2003) 555-562.

[7] M. Shabir, M. Naz, On soft topological spaces, Comput. Math. Appl. 61(2011) 1786-1799.

[8] S. Hussain and B. Ahmad, Some properties of soft topological spaces, Comput. Math. Appl. 62 (2011) 4058-4067.

[9] N. Cagman, S. Karatas and S. Enginoglu, Soft topology, Comput. Math. Appl. 62 (2011), 351-358.

[10] W. K. Min, A note on soft topological spaces, Comput. Math. Appl. 62 (2011) 3524-3528.

[11] J.C. Kelly, Bitopological Spaces, proc. London Math. Soc; 13 (1963) 71-83.

[12] S. Lal, Pairwise concepts in bitopological spaces, J. Aust. Math. Soc. (Ser.A), 26 (1978) 241-250.

[13] E. P. Lane, Bitopological spaces and quasi-uniform spaces, Proc. London Math. Soc. 17 (1967) 241-256.

[14] C. W. Patty, Bitopological spaces, Duke Math. J. 34 (1967) 387-392.
[15] W. J. Pervin, Connectedness in bitopological spaces, Indag. Math. 29 (1967) 369-372.

[16] I. L. Reilly, On bitopological separation properties, Nanta Math. 5 (1972) 14-25.

[17] M.K. Singal, Asha Rani Singal, Some more separation axioms in bitopological spaces, Ann.Soc. Sci. Bruxelles, 84 (1970) 207-230.

[18] Basavaraj M. Ittanagi, Soft Bitopological Spaces, Comp. and Math. with App., 107 (2014) 1-4.

[19] G. Senel, The Theory of Soft Ditopological Spaces, International Journal of Computer Applications, 150 (2016) 0975 - 8887.

[20] T. S. Dizman, A. Sostak, S. Yuksel, Soft Ditopological Spaces, Filomat 30:1, (2016) 209-222.

[21] M. Kovar, On 3-Topological version of Thet- Reularity, Internat.J. Matj, Sci., 23 (2000) 393- 398

[22] A. Luay, Tritopological spaces, Journal of Babylon University, 9 (2003) 33-45.

[23] Asmhan F. H., $\delta^{*}$-open set in tritopological spaces, M.Sc. thesis, Kufa University. (2004).

[24] F. Feng, Y.B. Jun, X.Z. Zhao, Soft semi rings, Computers and Math. with Appl. 56 (2008) 2621-2628.

[25] G. Senel, N. Çagman, Soft closed sets on soft bitopological space. Journal of new results in science, 5 (2014) 57-66. 